\title{
Solution of the Linear Programming Problems based on Neural Network Approach
}

\author{
Neeraj Sahu \\ Department of Mathematics and \\ Allied science Jiwaji University \\ Gwalior(M. P.), India,
}

\author{
Avanish Kumar \\ Department of Mathematical, \\ Sciences and Computer Applications, \\ Bundelkhand University \\ Jhansi (U. P.), India,
}

\begin{abstract}
In this paper we discuss solution of Linear Programming problems through neural network. Without having location restriction this network uses only simple hardware. Here we proved to be completely stable to exact solution without any multipliers. Moreover using this network we can solve linear programming problems and its dual simultaneously. These linear programming problems use circuit implementation.
\end{abstract}

\section{Keywords}

Neural network; Globally exponentially stable; Linear programming.

\section{INTRODUCTION}

Hopfield and Tank proposed a recurrent neural network for solving linear programming problems [1]. Sudharsanan and Sundareshan [2] introduced a method to programming a single layered recurrent neural network to execute optimization via quadratic minimization. The optimization method employed by generalized network is shown to descend in to the usual class of Gradient method for restrained nonlinear optimization. In contrast with penalty function method [3]. The optimization variables problem which can get up in such various topics as rigid body Mechanics, Fluid dynamics and elastic-plastic torsion [4]. The global exponential stability of different neural networks have been deliberate [5] in which weight matrices need to be accepted some sufficient conditions. The optimization of the new Back-Propagation algorithm by using imitative information [9].

Our work inspired Youshen Xia [6] and Youshen Xia, Jun Wang [7] Here we use neural network method for solving different type of linear programming problems. We use basic problem for mathematical formulation to extend a new network. Here we describe circuit implementation of proposed network for proportional analysis.

\section{LINEAR PROGRAMMING PROBLEMS OF THE GENERAL FORM}

The general form of the linear programming problems, that is considered for the solution purpose is an mentioned below;

$$
\begin{array}{cl}
\text { Minimize, } \quad c^{\mathrm{T}} \mathrm{x}+\mathrm{d}^{\mathrm{T}} \mathrm{x} \\
\text { Subject to: } & \mathrm{A}_{11} \mathrm{x}_{\mathrm{I}}+\mathrm{A}_{12} \mathrm{x}_{\mathrm{II}}+\mathrm{A}_{13} \mathrm{x}_{\mathrm{III}}+\mathrm{A}_{14} \mathrm{x}_{\mathrm{IV}} \geq \mathrm{b}_{\mathrm{I}} \\
& \mathrm{A}_{21} \mathrm{x}_{\mathrm{I}}+\mathrm{A}_{22} \mathrm{x}_{\text {II }}+\mathrm{A}_{23} \mathrm{x}_{\text {III }}+\mathrm{A}_{24} \mathrm{x}_{\mathrm{IV}} \geq \mathrm{b}_{\mathrm{II}}
\end{array}
$$

$$
\begin{aligned}
& \mathrm{A}_{31} \mathrm{x}_{\mathrm{I}}+\mathrm{A}_{32} \mathrm{x}_{\mathrm{II}}+\mathrm{A}_{33} \mathrm{x}_{\mathrm{III}}+\mathrm{A}_{34} \mathrm{x}_{\mathrm{IV}} \geq \mathrm{a}_{\mathrm{I}} \\
& \mathrm{A}_{41} \mathrm{x}_{\mathrm{I}}+\mathrm{A}_{42} \mathrm{x}_{\mathrm{II}}+\mathrm{A}_{43} \mathrm{x}_{\mathrm{III}}+\mathrm{A}_{44} \mathrm{x}_{\mathrm{IV}}=\mathrm{a}_{\mathrm{II}}
\end{aligned}
$$

$$
\mathrm{x} \geq 0
$$

Where,

$$
\begin{gathered}
x=\left(\begin{array}{l}
x_{I} \\
x_{I I} \\
x_{x_{I I}}
\end{array}\right) \in R^{n}, \quad c=\left(\begin{array}{c}
c_{I} \\
c_{I I}
\end{array}\right) \in R^{m}, \quad d=\left(\begin{array}{c}
d_{I} \\
d_{I I}
\end{array}\right) \in R^{m}, \\
a=\left(\begin{array}{c}
a_{I I} \\
a_{I I}
\end{array}\right) \in R^{m}, b=\left(\begin{array}{c}
b_{I I} \\
b_{I I}
\end{array}\right) \in R^{m}
\end{gathered}
$$

By the duality theory [7] the dual problem of the primal problem (1) is of the following form;

$$
\text { Maximize, } \quad b^{T} x+a^{T} x
$$

$$
\begin{aligned}
& \text { Subject to: } \mathrm{A}^{\mathrm{T}}{ }_{11} \mathrm{y}_{\mathrm{I}}+\mathrm{A}^{\mathrm{T}}{ }_{21} \mathrm{y}_{\mathrm{II}}+\mathrm{A}^{\mathrm{T}}{ }_{31} \mathrm{y}_{\mathrm{III}}+\mathrm{A}^{\mathrm{T}}{ }_{41} \mathrm{y}_{\mathrm{IV}} \leq \mathrm{c}_{\mathrm{I}} \\
& A^{\mathrm{T}}{ }_{12} \mathrm{y}_{\mathrm{I}}+\mathrm{A}^{\mathrm{T}}{ }_{22} \mathrm{y}_{\mathrm{II}}+\mathrm{A}^{\mathrm{T}}{ }_{32} \mathrm{y}_{\text {III }}+\mathrm{A}^{\mathrm{T}}{ }_{42} \mathrm{y}_{\mathrm{IV}} \leq \mathrm{c}_{\mathrm{II}} \\
& \mathrm{A}^{\mathrm{T}}{ }_{13} \mathrm{y}_{\mathrm{I}}+\mathrm{A}^{\mathrm{T}}{ }_{23} \mathrm{y}_{\text {II }}+\mathrm{A}^{\mathrm{T}}{ }_{33} \mathrm{y}_{\text {III }}+\mathrm{A}^{\mathrm{T}}{ }_{43} \mathrm{y}_{\text {IV }} \leq \mathrm{d}_{\mathrm{I}} \\
& \mathrm{A}^{\mathrm{T}}{ }_{14} \mathrm{y}_{\mathrm{I}}+\mathrm{A}^{\mathrm{T}}{ }_{24} \mathrm{y}_{\text {II }}+\mathrm{A}^{\mathrm{T}}{ }_{34} \mathrm{y}_{\text {III }}+\mathrm{A}^{\mathrm{T}}{ }_{44} \mathrm{y}_{\text {IV }}=\mathrm{d}_{\text {II }}
\end{aligned}
$$

$$
\mathrm{y} \geq 0
$$

Where, $\quad \mathrm{y}=\left(\begin{array}{c}\mathrm{y}_{\mathrm{II}} \\ y_{\mathrm{II}} \\ y_{\mathrm{y}_{\mathrm{IIV}}}\end{array}\right) \in \mathrm{R}^{\mathrm{m}}$

Both the special type cases (1) and (2) are of the following linear programming problem in its standard forms;

$$
\begin{array}{ll}
\text { Minimize, } & \mathrm{c}^{\mathrm{T}} \mathrm{x}+\mathrm{d}^{\mathrm{T}} \mathrm{x} \\
\text { Subject to: } & \mathrm{Ax} \geq \mathrm{a} \\
& \mathrm{Ax} \geq \mathrm{b} \\
& \mathrm{x} \geq 0
\end{array}
$$

Where, $A \in R^{m \times n}, c, d \in R^{n}$ and $a, b \in R^{m}$ and its dual 
Maximize, $\quad b^{T} x+a^{T} x$

Subject to: $\mathrm{A}^{\mathrm{T}} \mathrm{y} \leq \mathrm{c}$

$$
\mathrm{A}^{\mathrm{T}} \mathrm{y} \leq \mathrm{d}
$$

By the duality of convex programming [7]. If $x^{*}$ is a solution of (1) then there exists $y^{*}$ such that $y^{*}$ is a solution of (2). Let,

$$
\mathrm{D}=\left\{\mathrm{u}^{*}=\left(\mathrm{x}^{*}, \mathrm{y}^{*}\right)^{\mathrm{T}} \mid \mathrm{x}^{*} \text { solves }(1) \mathrm{y}^{*} \text { solve }(2)\right\}
$$

then a necessary and sufficient condition for $u=(x, y)^{T} \in D$ is following

$$
c^{T} x+d^{T} x=b^{T} x+a^{T} x
$$

$\left(A_{11}, A_{12}, A_{13}, A_{14}\right) x \geq b_{I}, \quad\left(A_{21}, A_{22}, A_{23}, A_{24}\right) x \geq b_{I}$

$\left(\mathrm{A}_{31}, \mathrm{~A}_{32}, \mathrm{~A}_{33}, \mathrm{~A}_{34}\right) \mathrm{x} \geq \mathrm{a}_{\mathrm{I}} \quad\left(\mathrm{A}_{41}, \mathrm{~A}_{42}, \mathrm{~A}_{43}, \mathrm{~A}_{44}\right) \mathrm{x}=\mathrm{a}_{\text {III }}$,

$\left(\mathrm{A}^{\mathrm{T}}{ }_{11}, \mathrm{~A}^{\mathrm{T}}{ }_{21}, \mathrm{~A}^{\mathrm{T}}{ }_{31}, \mathrm{~A}^{\mathrm{T}}{ }_{41}\right) \mathrm{y} \leq \mathrm{c}_{\mathrm{I}}, \quad\left(\mathrm{A}^{\mathrm{T}}{ }_{12}, \mathrm{~A}^{\mathrm{T}}{ }_{22}, \mathrm{~A}^{\mathrm{T}}{ }_{32}, \mathrm{~A}^{\mathrm{T}}{ }_{42}\right) \mathrm{y} \leq \mathrm{c}_{\text {II }}, \quad$ (5)

$\left(\mathrm{A}^{\mathrm{T}}{ }_{13}, \mathrm{~A}^{\mathrm{T}}{ }_{23}, \mathrm{~A}^{\mathrm{T}}{ }_{33}, \mathrm{~A}^{\mathrm{T}}{ }_{43}\right) \mathrm{y} \leq \mathrm{d}_{\mathrm{I}},\left(\mathrm{A}^{\mathrm{T}}{ }_{14}, \mathrm{~A}^{\mathrm{T}}{ }_{24}, \mathrm{~A}^{\mathrm{T}}{ }_{34}, \mathrm{~A}^{\mathrm{T}}{ }_{44}\right) \mathrm{y}=\mathrm{d}_{\text {II }}$

Thus we define,

$\mathrm{E}(\mathrm{x}, \mathrm{y})=\frac{1}{2}\left(\mathrm{c}^{\mathrm{T}} \mathrm{x}-\mathrm{b}^{\mathrm{T}} \mathrm{y}\right)^{2}+\frac{1}{2}\left(\mathrm{~d}^{\mathrm{T}} \mathrm{x}-\mathrm{y}\right)^{2}+\frac{1}{2} \mathrm{x}_{\mathrm{I}}{ }^{\mathrm{T}}\left(\mathrm{x}_{\mathrm{I}}-\| \mathrm{x}_{\mathrm{I}} \mathrm{I}\right)+\frac{1}{2} \mathrm{y}_{\mathrm{I}}^{\mathrm{T}}\left(\mathrm{y}_{\mathrm{I}}-\mathrm{Iy}_{\mathrm{I}} \mathrm{l}\right)$

$+\frac{1}{2}\left\|A_{2} x-b_{I I}\right\|_{2}^{2}+\frac{1}{2}\left\|A_{4} y-c_{I I}\right\|_{2}^{2}+\frac{1}{2}\left\|A_{6} x-a_{I I}\right\|_{2}^{2}+$

$\left\|A_{g} y-d_{I I}\right\|_{2}^{2}+\frac{1}{2}\left[A_{1} x-b_{1}\right]^{T}\left[\left(A_{1} x-b_{1}\right)-\| A_{1} x-b_{1} \rrbracket\right]$

$+\frac{1}{2}\left[-A_{a} y+c_{1}\right]^{T}\left[\left(-A_{a} y+c_{1}\right)-\left\|-A_{a} y+c_{1}\right\|\right]$

$+\frac{1}{2}\left[A_{5} x-a_{1}\right]^{T}\left[\left(A_{5} x-a_{1}\right)-\left\|A_{5} x-a_{1}\right\|\right]$

$+\frac{1}{2}\left[-A_{7} y+d_{1}\right]^{T}\left[\left(-A_{7} y+d_{1}\right)-1-A_{7} y+d_{1} I\right]$

Where,

$\mathrm{A}_{1}=\left(\mathrm{A}_{11}, \mathrm{~A}_{12}, \mathrm{~A}_{13}, \mathrm{~A}_{14}\right), \quad \mathrm{A}_{2}=\left(\mathrm{A}_{21}, \mathrm{~A}_{22}, \mathrm{~A}_{23}, \mathrm{~A}_{24}\right)$,

$\mathrm{A}_{3}=\left(\mathrm{A}_{31}, \mathrm{~A}_{32}, \mathrm{~A}_{33}, \mathrm{~A}_{34}\right), \quad \mathrm{A}_{4}=\left(\mathrm{A}_{41}, \mathrm{~A}_{42}, \mathrm{~A}_{43}, \mathrm{~A}_{44}\right)$,

$\mathrm{A}_{5}=\left(\mathrm{A}_{11}^{\mathrm{T}}, \mathrm{A}^{\mathrm{T}}{ }_{21}, \mathrm{~A}^{\mathrm{T}}{ }_{31}, \mathrm{~A}^{\mathrm{T}}{ }_{41}\right), \mathrm{A}_{6}=\left(\mathrm{A}_{12}^{\mathrm{T}}, \mathrm{A}^{\mathrm{T}} 22, \mathrm{~A}^{\mathrm{T}}{ }_{32}, \mathrm{~A}^{\mathrm{T}}{ }_{42}\right)$

$\mathrm{A}_{7}=\left(\mathrm{A}^{\mathrm{T}}{ }_{13}, \mathrm{~A}^{\mathrm{T}}{ }_{23}, \mathrm{~A}^{\mathrm{T}} 33, \mathrm{~A}^{\mathrm{T}}{ }_{43}\right), \mathrm{A}_{8}=\left(\mathrm{A}^{\mathrm{T}}{ }_{14}, \mathrm{~A}^{\mathrm{T}}{ }_{24}, \mathrm{~A}^{\mathrm{T}}{ }_{34}, \mathrm{~A}^{\mathrm{T}}{ }_{44}\right)$

And $\mid x \in\left(\left|x_{1}\right| \ldots \ldots\left|x_{n}\right|\right)^{T}$, then it is easy to see that a necessary and sufficient condition for $(\mathrm{x}, \mathrm{y})^{\mathrm{T}} \in \mathrm{D}$ can be expressed as $\mathrm{E}(\mathrm{x}, \mathrm{y})=0$

\section{CIRCUIT IMPLEMENTATION OF THE NETWORK}

Consider new neural network for solving (1) and (2) whose state vector $\mathrm{u}=(\mathrm{x}, \mathrm{y})^{\mathrm{T}}$ is governed by following differential equation

$$
\frac{\mathrm{du}}{\mathrm{dt}}=-\nabla \mathrm{E}(\mathrm{u}), \quad \mathrm{u} \in \mathrm{R}^{\mathrm{n}+\mathrm{m}}
$$

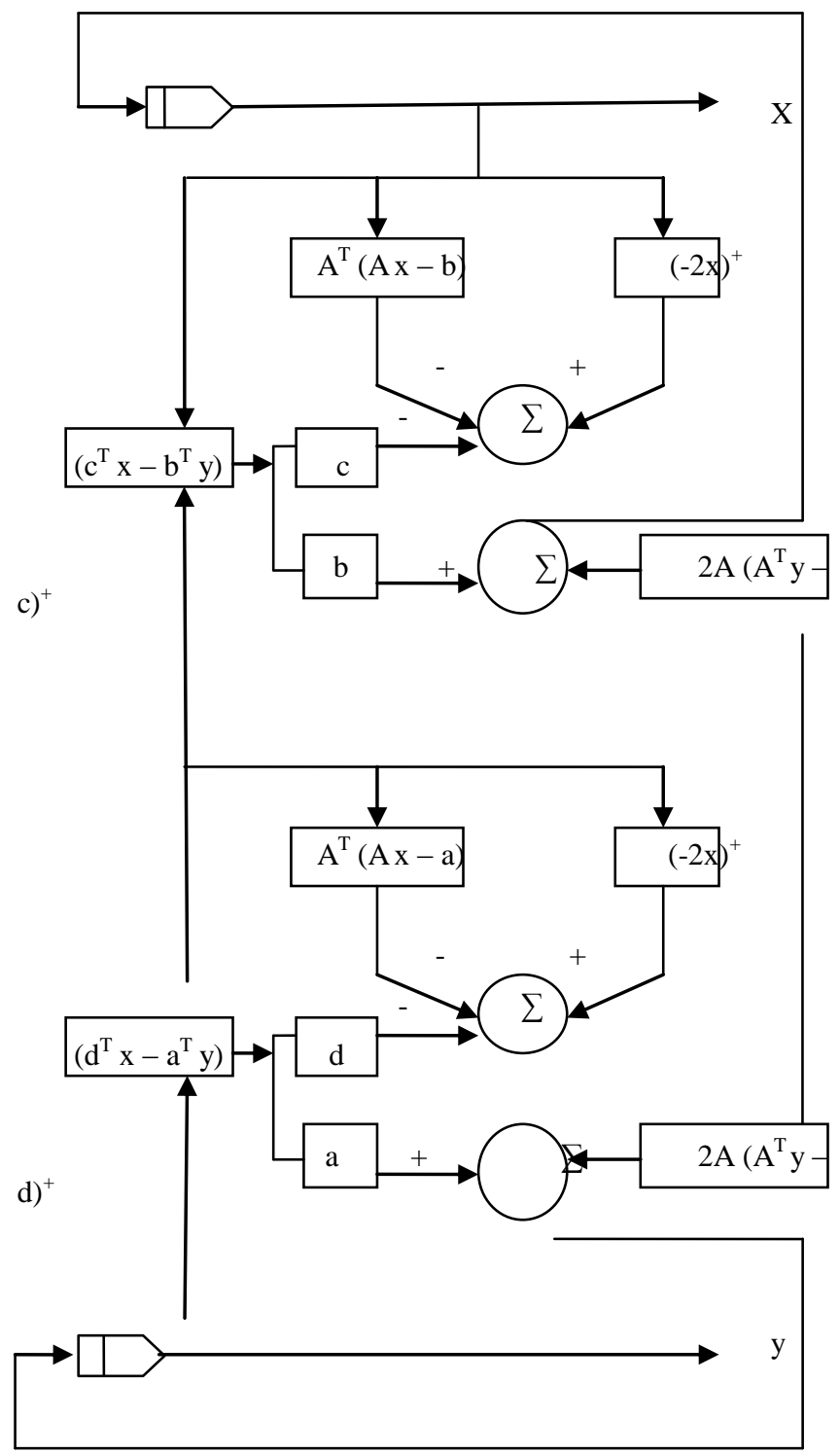

Where $\nabla_{\mathrm{E}}(\mathrm{u})$ is the gradient of energy function $\mathrm{E}(\mathrm{u})$ defined as in section 2 .

We know that $\mathrm{x}-|\mathrm{x}|=2(\mathrm{x})^{-}$, then the dynamics of projected network in (6) is represented as,

$\frac{d x_{I}}{d t}=\left\{\left(c^{T} x-b^{T} y\right) c_{I}+2\left(x_{I}\right)^{-}+A_{21}^{T}\left(A_{2} x-b_{I I}\right)+2 A_{11}^{T}\left(A_{1} x-\right.\right.$

$\left.\left.\mathrm{b}_{1}\right)^{-}\right\}+\left\{\left(\mathrm{d}^{\mathrm{T}} \mathrm{x}-\mathrm{a}^{\mathrm{T}} \mathrm{y}\right) \mathrm{c}_{\mathrm{I}}+2\left(\mathrm{x}_{\mathrm{I}}\right)^{-}+\mathrm{A}_{23}{ }^{\mathrm{T}}\left(\mathrm{A}_{2} \mathrm{x}-\mathrm{b}_{\text {II }}\right)+2 \mathrm{~A}_{13}{ }^{\mathrm{T}}\left(\mathrm{A}_{1} \mathrm{x}-\right.\right.$ $\left.\left.\mathrm{b}_{1}\right)^{-}\right\}$

$\frac{d x_{I I I}}{d t}=\left\{\left(c^{T} x-b^{T} y\right) d_{I}+2\left(x_{I}\right)^{-}+A_{41}{ }^{T}\left(A_{4} x-a_{I I}\right)+2 A_{31}{ }^{T}\left(A_{3} x\right.\right.$ $\left.\left.-\mathrm{a}_{1}\right)^{-}\right\}+\left\{\left(\mathrm{d}^{\mathrm{T}} \mathrm{x}-\mathrm{a}^{\mathrm{T}} \mathrm{y}\right) \mathrm{d}_{\mathrm{I}}+2\left(\mathrm{x}_{\mathrm{I}}\right)^{-}+\mathrm{A}_{43}^{\mathrm{T}}\left(\mathrm{A}_{4} \mathrm{x}-\mathrm{a}_{\mathrm{II}}\right)+2 \mathrm{~A}_{33}{ }^{\mathrm{T}}\left(\mathrm{A}_{3} \mathrm{X}-\right.\right.$ $\left.\left.a_{1}\right)^{-}\right\}$ 


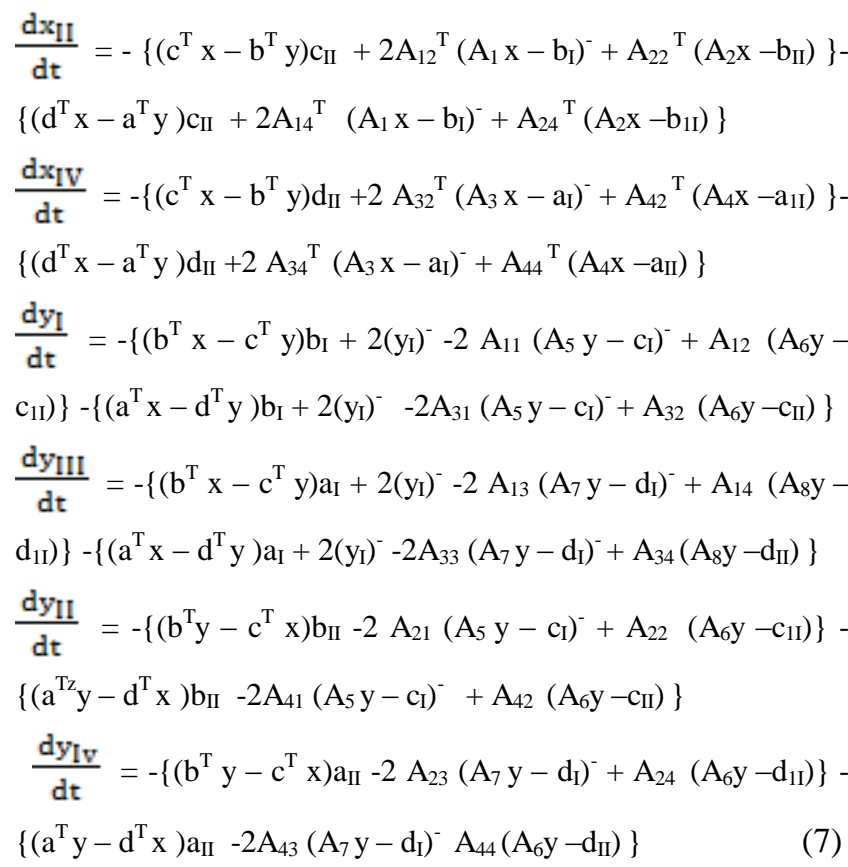

Where, $(x)^{-}=\left(x_{1}^{-}, x_{2}^{-}, \ldots . x_{n}^{-}\right)^{T}, x_{i}^{-}=\min \left\{0, x_{i}\right\}$ and $A_{1}, A_{2}, A_{3}$, $A_{4}, A_{5}, A_{6}, A_{7}, A_{8}$ are defined in section 2. Here this network uses only simple hardware and non of the multipliers and dividers are necessary. For expediency, we only give the network circuit implementation for solving (3) and (4) whose dynamics are governed by the following,

$\frac{d x}{d t}=-\left\{\left(c^{T} x-b^{T} y\right) c+2(x)^{-}+A^{T}(A x-b)\right\}-\left\{\left(d^{T} x-a^{T} y\right) d+\right.$ $\left.2(\mathrm{x})^{-}+\mathrm{A}^{\mathrm{T}}(\mathrm{Ax}-\mathrm{b})\right\}$

$\frac{d y}{d t}=-\left\{\left(b^{T} y-c^{T} x\right) b-2 A\left(c-A^{T} y\right)^{-}\right\}-\left\{\left(a^{T} y-d^{T} x\right) a-2 A\right.$

$\left.\left(\mathrm{d}-\mathrm{A}^{\mathrm{T}} \mathrm{y}\right)^{-}\right\}$

Note that $(x)^{-}=-(-x)^{+}$, then (8) may be restated as,

$\frac{d x}{d t}=-\left\{\left(c^{T} x-b^{T} y\right) c-(-2 x)^{+}+A^{T}(A x-b)\right\}-\left\{\left(d^{T} x-a^{T} y\right) d-\right.$

$\left.(-2 x)^{+}+A^{T}(A x-b)\right\}$

$\frac{d y}{d t}=\left(c^{T} x-b^{T} y\right) b-2 A\left(A^{T} y-c\right)^{+}+\left(d^{T} x-a^{T} y\right) a-2 A\left(A^{T} y-\right.$ d) $\left.{ }^{+}\right\}$

Where, $(\mathrm{x})^{+}=\left|\left(\mathrm{x}_{1}\right)^{+}, \ldots \ldots\left(\mathrm{x}_{\mathrm{n}}\right)^{+}\right|^{\mathrm{T}}\left(\mathrm{x}_{\mathrm{i}}\right)^{+}=\max \left\{0, \mathrm{x}_{\mathrm{i}}\right\}$, a simplified network diagram of (9) which is shown in figure where vector $\mathrm{a}, \mathrm{b}, \mathrm{c}, \mathrm{d}$ are external input and $\mathrm{x}, \mathrm{y}$ are of the network out put.

\section{THEOREM}

Assume that (3) and (4) have unique optimal solution. Then the primal dual neural network in given function $\mathrm{F}(\mathrm{Z})$ is globally exponentially stable i.e;

$$
\begin{aligned}
& \frac{d V(z)}{d t}=-F(Z)=\quad\left\{\left(c^{T} x-b^{T} y\right) c-(-x)^{+}+A^{T}(A x-b)\right. \\
& \left(d^{T} x-a^{T} y\right) d-(-x)^{+} A^{T}(A x-a)-\left(c^{T} x-b^{T} y\right) b+A\left(A^{T} y-c\right)^{+} \\
& \left.-\left(d^{T} x-a^{T} y\right) a+A\left(A^{T} y-d\right)^{+}\right\}
\end{aligned}
$$

Proof : Because (3) and (4) have unique optimal solution of system (10) that has unique equilibrium point. Let $\mathrm{Z}^{*}=\left(\mathrm{x}^{*}, \mathrm{y}^{*}\right)$ be an optimal solution to (3) and (4) and $\mathrm{V}(\mathrm{Z})=\left\|\mathrm{Z}-\mathrm{Z}^{*}\right\|^{\mathbf{2}} / 2$

$\frac{d v(z)}{d t}=-\left\{\begin{array}{l}x-x \\ y-y^{*}\end{array}\right\} F(z)$

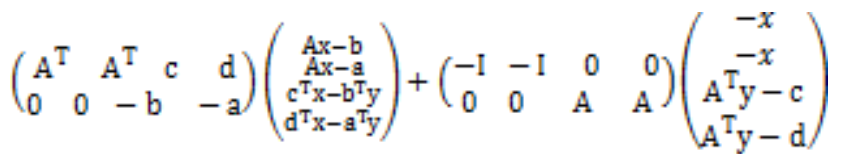

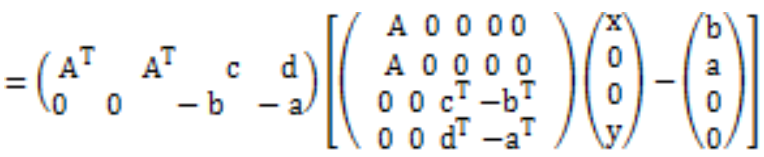

$+\left(\begin{array}{cccc}-\mathrm{I} & -\mathrm{I} & 0 & 0 \\ 0 & 0 & \mathrm{~A} & \mathrm{~A}\end{array}\right)\left[\left(\begin{array}{cccc}-\mathrm{I} & -\mathrm{I} & 0 & 0 \\ 0 & 0 & \mathrm{~A}^{\mathrm{T}} & \mathrm{A}^{\mathrm{T}}\end{array}\right)\left(\begin{array}{l}x \\ x \\ y \\ y\end{array}\right)-\left(\begin{array}{l}0 \\ 0 \\ c \\ d\end{array}\right)\right]$

$=\left\{\begin{array}{lll}x-x^{*} \\ y-y^{*}\end{array}\right\}^{T}\left(\begin{array}{cccc}A^{T} & A^{T} & c & d \\ 0 & 0 & -b & -a\end{array}\right)\left[\left(\begin{array}{ccccc}A & 0 & 0 & 0 & 0 \\ A & 0 & 0 & 0 & 0 \\ 0 & 0 & c^{T} & -b^{T} \\ 0 & 0 & d^{T} & -a^{T}\end{array}\right)\left(\begin{array}{l}x \\ 0 \\ 0 \\ y\end{array}\right)-\left(\begin{array}{l}b \\ a \\ 0 \\ 0\end{array}\right)\right]$

$=\left\|\left(\begin{array}{ccccc}A & 0 & 0 & 0 & 0 \\ A & 0 & 0 & 0 & 0 \\ 0 & 0 & c^{T} & -b^{T} \\ 0 & 0 & d^{T} & -a^{T}\end{array}\right)\left(\begin{array}{l}x \\ 0 \\ 0 \\ y\end{array}\right)-\left(\begin{array}{l}b \\ a \\ 0 \\ 0\end{array}\right)\right\|^{2}$

And

$$
\begin{aligned}
& \left\{\begin{array}{l}
\mathrm{x}-\mathrm{x}^{2} \\
\mathrm{y}-\mathrm{y}^{0}
\end{array}\right\}^{\mathrm{T}}\left(\begin{array}{cccc}
-\mathrm{I} & -\mathrm{I} & 0 & 0 \\
0 & 0 & \mathrm{~A} & \mathrm{~A}
\end{array}\right)\left[\left(\begin{array}{cccc}
-\mathrm{I} & -\mathrm{I} & 0 & 0 \\
0 & 0 & \mathrm{~A}^{\mathrm{T}} & \mathrm{A}^{\mathrm{T}}
\end{array}\right)\left(\begin{array}{l}
x \\
x \\
y \\
y
\end{array}\right)-\left(\begin{array}{l}
0 \\
0 \\
c \\
d
\end{array}\right)\right] \\
& =\left\|\left(\begin{array}{cccc}
-\mathrm{I} & -\mathrm{I} & 0 & 0 \\
0 & 0 & \mathrm{~A}^{\mathrm{T}} & \mathrm{A}^{\mathrm{T}}
\end{array}\right)\left(\begin{array}{l}
x \\
x \\
y \\
y
\end{array}\right)-\left(\begin{array}{l}
0 \\
0 \\
c \\
d
\end{array}\right)\right\|^{2}
\end{aligned}
$$

Thus

$=\left\{\begin{array}{ll}x-x^{2} \\ y-y^{*}\end{array}\right\}^{T} F(Z) \geq\left\|\left(\begin{array}{ccccc}A & 0 & 0 & 0 & 0 \\ A & 0 & 0 & 0 & 0 \\ 0 & 0 & c^{T} & -b^{T} \\ 0 & 0 & d^{T} & -a^{T}\end{array}\right)\left(\begin{array}{l}x \\ 0 \\ 0 \\ y\end{array}\right)-\left(\begin{array}{l}b \\ a \\ 0 \\ 0\end{array}\right)\right\|^{2}$ 


$$
+\left\|\left(\begin{array}{cccc}
-\mathrm{I} & -\mathrm{I} & 0 & 0 \\
0 & 0 & \mathrm{~A}^{\mathrm{T}} & \mathrm{A}^{\mathrm{T}}
\end{array}\right)\left(\begin{array}{l}
x \\
x \\
y \\
y
\end{array}\right)-\left(\begin{array}{l}
0 \\
0 \\
c \\
d
\end{array}\right)\right\|^{2}
$$

From the well known Hoffman inequality [8]. It follows,

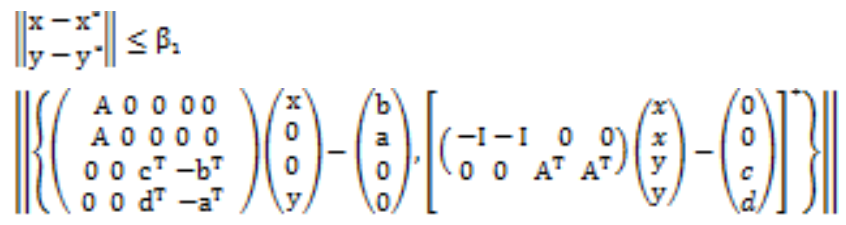

Then

$\left\|\begin{array}{l}x-x^{*} \\ y-y^{*}\end{array}\right\|^{*} \leq$

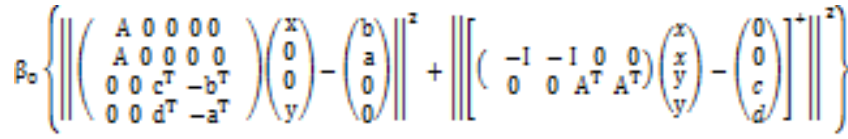

When $\beta_{1}>0$ and $\beta_{0}=\beta_{1}{ }^{2}$ thus $\frac{\mathrm{d}}{\mathrm{dt}} \mathrm{V}(\mathrm{Z}) \leq-2 \beta_{0}{ }^{-1} \mathrm{~V}(\mathrm{Z})$

Hence $\left\|z(t)-z^{*}\right\| \leq\left\|z\left(t_{0}\right)-z^{*}\right\| \quad e^{2 \beta_{0}^{-1}\left(t-t_{0}\right)}$, so the primal dual neural network (10) is globally exponentially stable.

\section{CONCLUSION}

Providing solution of the linear programming problems through neural network approach is an interesting area of research. The research suggested in this paper is defining an energy function of the linear programming and its dual problem. A circuit has been designed for the purpose. A new neural network is discussed in circuit implementation. Further, in this paper we have proved that the primal dual neural network is globally exponentially stable. It is useful for solving many optimization and related problems.

\section{REFERENCES}

[1] Tank, D. W. and Hopfield, J. J., 1986. "Simple neural optimization network: An A/D converter, singal decision circuit, and a linear programming circuit", IEEE Trans. Circuit syst., Vol.33, pp.533-541.

[2] Sudharsanan, S. and Sundareshan, M., 1991. "Exponential stability and a systematic of neural network for quadratic minimization", Neural networks Vol. 4 no.5, pp.599-613.

[3] Bazaraa, M. S. and Shetty, C., 1979. "Nonlinear programming: Theory and Algorithms", New York: Wiley.

[4] More, J. J. and Toraldo G., 1991. "On the solution of large quadratic programming problems without bounded constraints”, SIAM J. Optimization, Vol.1, no.1, pp.93-113.

[5] Michel, A. N. Farrell, J. A. and Porod, W., 1989. "Qualitative analysis of neural network", IEEE Trans. Circuit Syst., Vol.36, pp.229-243.

[6] Xia, Youshen., March 1986. "A new neural network for solving linear programming problems and application", IEEE Trans. Neural Network, Vol.7, no.2, pp.525-529.

[7] Xia, Youshen. and Wang, Jun., July 2000. "Global Exponential stability of recurrent neural network for solving Optimization related problems", IEEE Trans. Neural Network, Vol.11, no.4, pp.1017-1022.

[8] Pang, J. S., 1987. "A posteriori error bounds for the linearlyconstrained variational inequality problems", Math. Oper. Res., Vol.12, pp.474-484.

[9] Zweiri, yahya H., 2007. "Optimization of a three Backpropagation Algorithm used for neural network learning", International Journal of Computational Intelligence 3;4 pp.322-327. 